University of Nebraska - Lincoln DigitalCommons@University of Nebraska - Lincoln

Uniformed Services University of the Health

Sciences

U.S. Department of Defense

2016

Health care worker follow-up compliance after occupational bloodborne pathogens exposure: A brief report

Juan C. Díaz

Uniformed Services University of the Health Sciences

Lucas A. Johnson

Uniformed Services University of the Health Sciences

Follow this and additional works at: http://digitalcommons.unl.edu/usuhs

Díaz, Juan C. and Johnson, Lucas A., "Health care worker follow-up compliance after occupational bloodborne pathogens exposure: A brief report" (2016). Uniformed Services University of the Health Sciences. 164.

http://digitalcommons.unl.edu/usuhs/164

This Article is brought to you for free and open access by the U.S. Department of Defense at DigitalCommons@University of Nebraska - Lincoln. It has been accepted for inclusion in Uniformed Services University of the Health Sciences by an authorized administrator of DigitalCommons@University of Nebraska - Lincoln. 


\title{
Health care worker follow-up compliance after occupational bloodborne pathogens exposure: A brief report
}

\author{
CPT Juan C. Díaz MC, USA *, LCDR Lucas A. Johnson MC, USN \\ Uniformed Services University of the Health Sciences, Bethesda, MD
}

Key Words:

Public health

Workplace injury

\begin{abstract}
A retrospective cohort study was conducted examining health care worker (HCW) compliance with Centers for Disease Control and Prevention recommendations following occupational bloodborne pathogen (BBP) exposure. HCWs with a BBP exposure from a known HIV- or hepatitis C virus-seropositive individual were less likely to complete recommended follow-up compared with HCWs with seronegative source patient exposures (adjusted odds ratio, 0.02 and 0.09, respectively). Continued targeted education and extra vigilance in performing postexposure surveillance are warranted in this higher-risk population.

Published by Elsevier Inc. on behalf of Association for Professionals in Infection Control and Epidemiology, Inc.
\end{abstract}

\section{BACIKGROUND}

Exposure to bloodborne pathogens (BBPs) is a well-described occupational hazard affecting an estimated 385,000 health care workers (HCWs) annually. ${ }^{1}$ Sharps injuries sustained by HCWs while providing patient care can potentially transmit more than 20 pathogens, most commonly hepatitis B virus (HBV), hepatitis C virus (HCV), and HIV. ${ }^{1}$ An estimated $8 \%$ of $\mathrm{HCV}, 1 \%$ of HBV, and $0.5 \%$ of HIV infections in HCWs are directly attributable to occupational sharps injuries $^{2}$; risk of seroconversion after percutaneous exposure to seropositive source patients is estimated at $6 \%-30 \%$ for $\mathrm{HBV}, 0.5 \%-2 \%$ for $\mathrm{HCV}$, and $0.3 \%$ for HIV.

The Centers for Disease Control and Prevention (CDC) recommends health care institutions implement postexposure protocols for injured HCWs, including assessment of source patients for evidence of HIV, HCV, and HBV infection as well as periodic followup testing and management of the injured HCW. ${ }^{3-5}$ Although previous studies demonstrate HCW compliance with performing CDCrecommended follow-up ranges between $33 \%$ and $87 \%$, none have systematically evaluated factors influencing HCW completion of follow-up. ${ }^{6,7}$

\footnotetext{
* Address correspondence to CPT Juan C. Díaz, MC, USA, Uniformed Services University of the Health Sciences, 4301 Jones Bridge Rd, Rm A-1040A, Bethesda, MD 20814.

E-mail address: juancdiazmd@icloud.com (J.C. Díaz).

Conflicts of Interest: None to report.
}

\section{METHODS}

A retrospective cohort study was designed using de-identified data routinely collected by the occupational medicine department of a large military treatment facility to evaluate clinical and demographic factors influencing HCW completion of CDCrecommended follow-up. Subjects were eligible for inclusion if they reported an occupational BBP exposure to the hospital's occupational medicine clinic or emergency room from August 1, 2011October 31, 2014. Hospital resident physicians and fellows sustaining offsite occupational exposures while performing a rotation at another facility were also eligible for inclusion because hospital policy mandated local administrative tracking and management of such exposures. The surveillance period was from August 1, 2011March 31, 2015, to allow subjects to complete a minimum of 6 months of follow-up after exposure. Primary outcome measure was completion of CDC-recommended management and followup testing defined as completion of all required postexposure laboratory studies at least once a minimum of 6 months after exposure.

Analysis of demographic and clinical covariate influence upon outcome was restricted only to those HCWs who had the opportunity to complete at least 6 months of follow-up after exposure. Two separate multivariate models were generated (1 evaluating influence of HIV source patient seropositivity and 1 for HCV) because inclusion of both HIV and HCV covariates into a single model resulted in zero cell counts and prohibited performance of logistic regression. This project was reviewed and approved by the Uniformed Services University of the Health Sciences' Office of Research (T0873293) and Walter Reed National Military Medical Center Institutional Review Board (408721-1). 


\section{RESULTS}

A total of 582 occupational BBP exposures were reported during the exposure period (Table 1 ). The majority of exposures involved source patients with no serologic evidence of $\operatorname{HIV}(73.6 \% ; n=428)$, $\operatorname{HBV}(75.3 \% ; n=438)$, or $\operatorname{HCV}(73.2 \% ; n=426)$.

\section{Table 1}

Characteristics of health care workers with reported bloodborne pathogens exposure, by completion of follow-up

\begin{tabular}{|c|c|c|c|c|}
\hline \multirow[b]{4}{*}{ Risk factor } & \multicolumn{3}{|c|}{$\begin{array}{l}\text { Completion of } \\
\text { follow-up* }\end{array}$} & \multirow[b]{4}{*}{$\chi^{2} P$ value } \\
\hline & \multirow{3}{*}{$\begin{array}{c}n(\%) \\
N=582\end{array}$} & Yes (\%) & No (\%) & \\
\hline & & $414(87.5)$ & $59(12.5)$ & \\
\hline & & \multicolumn{2}{|c|}{ Total 473} & \\
\hline Employment status & & & & .08 \\
\hline Active duty & $436(74.9)$ & $310(87.3)$ & $45(12.7)$ & \\
\hline Civilian & $45(7.7)$ & $40(93.0)$ & $3(7.0)$ & \\
\hline Contractor & $66(11.3)$ & $45(86.5)$ & $7(13.5)$ & \\
\hline General schedule & $34(5.8)$ & $19(86.4)$ & $3(13.6)$ & \\
\hline Unknown & $1(.2)$ & $0(0.0)$ & $1(100.0)$ & \\
\hline Institution role & & & & .01 \\
\hline Medical student & $23(4.0)$ & $17(94.4)$ & $1(5.6)$ & \\
\hline Midlevel provider & $19(3.3)$ & $14(87.5)$ & $2(12.5)$ & \\
\hline Nurse & $124(21.3)$ & 91 (91.9) & $8(8.1)$ & \\
\hline Physician & $161(27.7)$ & $127(90.1)$ & $14(9.9)$ & \\
\hline Medical technician & $201(34.5)$ & $129(83.8)$ & $25(16.2)$ & \\
\hline Other & $54(9.3)$ & $36(80.0)$ & $9(20.0)$ & \\
\hline Department & & & & .02 \\
\hline Dental & $34(5.8)$ & $24(88.9)$ & $3(11.1)$ & \\
\hline Fire/police & $3(.5)$ & $1(33.3)$ & $2(66.7)$ & \\
\hline Housekeeping & $21(3.6)$ & $14(82.4)$ & $3(17.6)$ & \\
\hline Medicine & $239(41.1)$ & $173(87.4)$ & $25(12.6)$ & \\
\hline Offsite & $17(2.9)$ & $11(73.3)$ & $4(26.7)$ & \\
\hline SPD & $25(4.3)$ & $15(75.0)$ & $5(25.0)$ & \\
\hline Surgery & $239(41.1)$ & $172(91.0)$ & $17(9.0)$ & \\
\hline Unknown & $4(.7)$ & $4(100.0)$ & $0(0.0)$ & \\
\hline Injury location & & & & $<.01$ \\
\hline Arm & $23(4.0)$ & $17(89.5)$ & $2(10.5)$ & \\
\hline Eye/face/mouth & $118(20.3)$ & $79(81.4)$ & $18(18.6)$ & \\
\hline Hand/finger & $423(72.7)$ & $310(90.4)$ & $33(9.6)$ & \\
\hline Leg & $9(1.5)$ & $4(66.7)$ & $2(33.3)$ & \\
\hline Other & $9(1.5)$ & $4(50.0)$ & $4(50.0)$ & \\
\hline Instrument type & & & & $<.01$ \\
\hline Blade & $42(7.2)$ & $32(94.1)$ & $2(5.9)$ & \\
\hline Hollow needle & $190(32.6)$ & $141(91.0)$ & $14(9.0)$ & \\
\hline Instrument & $77(13.2)$ & $51(85.0)$ & $9(15.0)$ & \\
\hline Needle, unknown type & $22(3.8)$ & $19(90.5)$ & $2(9.5)$ & \\
\hline Solid needle & $126(21.6)$ & $97(94.2)$ & $6(5.8)$ & \\
\hline Splash & $114(19.6)$ & $65(73.0)$ & $24(27.0)$ & \\
\hline Other & $11(1.9)$ & $9(81.8)$ & $2(18.2)$ & \\
\hline Source HIV rapid test & & & & $<.01$ \\
\hline Negative & $428(73.6)$ & $338(96.8)$ & $11(3.2)$ & \\
\hline Positive & $17(2.9)$ & $6(37.5)$ & $10(62.5)$ & \\
\hline Unknown & $137(23.5)$ & $70(64.8)$ & $38(35.2)$ & \\
\hline Source hepatitis C status & & & & $<.01$ \\
\hline Negative & $426(73.2)$ & $337(97.4)$ & $9(2.6)$ & \\
\hline Positive & $14(2.4)$ & $7(50.0)$ & $7(50.0)$ & \\
\hline Unknown & $142(24.4)$ & $70(61.9)$ & $43(38.1)$ & \\
\hline Source hepatitis B status & & & & .43 \\
\hline Negative & $438(75.3)$ & $341(95.3)$ & $17(4.7)$ & \\
\hline Positive & $3(.5)$ & $3(100.0)$ & $0(0.0)$ & \\
\hline Unknown & $141(24.2)$ & $70(62.5)$ & $42(37.5)$ & \\
\hline $\begin{array}{l}\text { Infectious diseases } \\
\text { department referral }\end{array}$ & & & & $<.01$ \\
\hline No & $537(92.3)$ & $392(90.1)$ & $43(9.9)$ & \\
\hline Yes & $45(7.7)$ & $22(57.9)$ & $16(42.1)$ & \\
\hline Placed on PEP & & & & .13 \\
\hline No & $17(37.8)$ & $7(46.7)$ & $8(53.3)$ & \\
\hline Yes & $28(62.2)$ & $15(65.2)$ & $8(34.8)$ & \\
\hline
\end{tabular}

PEP, post exposure prophylaxis.

*Completion of follow-up restricted to subjects with at least 6 months of observation after exposure (August 1,2011-March 31, 2014).
Of the 473 subjects whose date of injury afforded a minimum of 6 months of observation, $88 \%(n=414)$ completed CDCrecommended follow-up. Institution role, department, injury location, instrument type, source HIV status, source HCV status, and infectious diseases department referral were significantly associated with the outcome on $\chi^{2}$ analysis.

On univariate analysis, HCWs with exposure to a known HIVseropositive source were $98 \%$ less likely to complete follow-up (odds ratio [OR], 0.02; 95\% confidence interval [CI], 0.01-0.07) when compared with subjects exposed to a HIV-negative source; these findings persisted after multivariate adjustment (adjusted OR [aOR], 0.02; 95\% CI, 0.004-0.13) (Table 2). Those exposed to source patients with unknown HIV serostatus demonstrated similarly decreased likelihood of follow-up (aOR, 0.03; 95\% CI, 0.01-0.08).

Source patient HCV serostatus similarly influenced likelihood of follow-up completion. On crude analysis, subjects with exposure to a known HCV-seropositive source patient were 97\% less likely to complete follow-up when compared with subjects exposed to a known seronegative HCV source (OR, 0.03; 95\% CI, 0.01-0.10); these findings persisted after multivariate adjustment (aOR, 0.09; $95 \% \mathrm{CI}$, 0.02-0.45). Again, those exposed to source patients with unknown HCV serostatus demonstrated decreased likelihood of follow-up (aOR, 0.02; 95\% CI, 0.01-0.06).

\section{DISCUSSION}

HCWs with a BBP exposure involving HIV or HCV seropositive or serostatus unknown patients were less likely to complete followup compared with HCWs exposed to seronegative source patients. At least 2 possible explanations exist for these surprising findings. First, the study design necessarily resulted in differential outcome assessment bias because a seronegative source exposure resulted in achieving outcome status with relative ease (essentially at time of presentation) compared with an HCW with an HIV- or HCVseropositive source exposure requiring 6 months of follow-up to achieve outcome status. A second possible explanation can be found in the health belief model, a psychological model evaluating behavior using the 3 constructs of threat perception, benefit perception, and perceived disadvantage. ${ }^{8}$ Per hospital protocol, HCWs exposed to known HIV or HCV patients received consultation with infectious diseases specialists who perform a review of source patient infectivity, including a review of source patient viral suppressive therapy and viral load (data unavailable to the hospital occupational medicine clinic). It is possible that follow-up compliance may be affected by an HCWs low-perceived susceptibility following delivery of these data during specialist assessment; however, the deidentified data source utilized for this study prevented retrospective record review to explore such a hypothesis.

Interpretation of this study is subject to several limitations. BBP exposures are historically underreported. Despite an institutional policy mandating self-reporting, undoubtedly some individuals chose not to report a BBP exposure. Additionally, exposed HCWs with a sophisticated understanding of BBP exposure risk may self-stratify, resulting in an overly complaint cohort more motivated to complete therapy. Several demographic factors (eg, age, sex, race, and assessment of personal protective equipment use) were not systematically recorded and were unavailable for analysis. Finally, the study design resulted in differential observation time for exposed HCWs based on source serostatus.

Postexposure programs are essential to sustaining a healthy workforce and preventing pathogen transmission. These study results highlight need for further research to more completely assess factors differentially influencing the personal motivations for complying with recommended follow-up after BBP exposures, particularly for patients with higher-risk exposures. 
Table 2

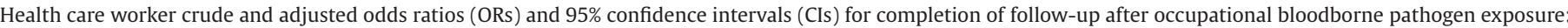
HIV and hepatitis C virus (HCV) models

\begin{tabular}{|c|c|c|c|c|c|c|}
\hline \multirow[b]{3}{*}{ Characteristics } & \multicolumn{3}{|c|}{ HIV model } & \multicolumn{3}{|c|}{ HCV model } \\
\hline & Crude OR & Adjusted model ${ }^{*}$ & & Crude odds ratio & Adjusted model ${ }^{*}$ & \\
\hline & OR (95\% CI) & OR (95\% CI) & $P$ value & OR (95\% CI) & OR (95\% CI) & $P$ value \\
\hline \multicolumn{7}{|l|}{ Institution role } \\
\hline Medical student & Reference category & Reference category & & Reference category & Reference category & \\
\hline Midlevel provider & $0.82(0.10-6.62)$ & $0.60(0.03-10.79)$ & .73 & $0.82(0.10-6.62)$ & $1.21(0.05-28.61)$ & .91 \\
\hline Nurse & $1.34(0.26-6.86)$ & $2.08(0.18-24.62)$ & .56 & $1.34(0.26-6.86)$ & $2.40(0.20-29.39)$ & .49 \\
\hline Physician & $1.07(0.22-5.11)$ & $1.44(0.13-16.49)$ & .77 & $1.07(0.22-5.11)$ & $1.28(0.11-14.93)$ & .85 \\
\hline Tech/corpsman/medic & $0.61(0.13-2.79)$ & $1.45(0.14-14.98)$ & .76 & $0.61(0.13-2.79)$ & $1.65(0.15-17.71)$ & .68 \\
\hline Other & $0.47(0.09-2.42)$ & $1.89(0.12-29.82)$ & .65 & $0.47(0.09-2.42)$ & 3.14 (0.19-53.29) & .43 \\
\hline \multicolumn{7}{|l|}{ Department } \\
\hline Surgery & Reference category & Reference category & & Reference category & Reference category & \\
\hline Fire/police & $0.05(0.01-0.60)$ & $0.09(0.01-2.37)$ & .15 & $0.05(0.01-0.60)$ & $0.41(0.003-61.39)$ & .73 \\
\hline Housekeeping & $0.49(0.13-1.86)$ & 1.73 (0.19-16.19) & 63 & $0.49(0.13-1.86)$ & $1.64(0.17-15.82)$ & .67 \\
\hline Medicine & $0.72(0.38-1.38)$ & $0.69(0.25-1.90)$ & .48 & $0.72(0.38-1.38)$ & $0.78(0.29-2.13)$ & .63 \\
\hline Offsite & $0.29(0.08-1.00)$ & $1.12(0.19-6.83)$ & .9 & $0.29(0.08-1.00)$ & $1.04(0.16-6.77)$ & .97 \\
\hline SPD & $0.31(0.10-0.97)$ & $2.07(0.42-10.24)$ & .37 & $0.31(0.10-0.97)$ & $2.37(0.47-12.05)$ & .3 \\
\hline Dental & $0.84(0.23-3.06)$ & $1.36(0.17-10.76)$ & .77 & $0.84(0.23-3.06)$ & $1.65(0.22-12.40)$ & .63 \\
\hline \multicolumn{7}{|l|}{ Injury location } \\
\hline Hand or finger & Reference category & Reference category & & Reference category & Reference category & \\
\hline Arm & $0.93(0.21-4.21)$ & $2.49(0.20-30.51)$ & .48 & $0.93(0.21-4.21)$ & $0.67(0.10-4.50)$ & .68 \\
\hline Eye/face/mouth & $0.48(0.26-0.90)$ & $1.44(0.29-7.15)$ & .66 & $0.48(0.26-0.90)$ & $1.43(0.26-7.89)$ & .68 \\
\hline Other & $0.11(0.03-0.46)$ & $0.14(0.01-1.56)$ & .11 & $0.11(0.03-0.46)$ & $0.18(0.02-2.06)$ & .17 \\
\hline Leg & $0.22(0.04-1.24)$ & $0.28(0.02-3.84)$ & .34 & $0.22(0.04-1.24)$ & $0.94(0.07-12.97)$ & .94 \\
\hline \multicolumn{7}{|l|}{ Instrument type } \\
\hline Solid needle & Reference category & Reference category & & Reference category & Reference category & \\
\hline Blade & $0.99(0.19-5.15)$ & $2.46(0.37-16.15)$ & .35 & $0.99(0.19-5.15)$ & $3.04(0.41-22.67)$ & .28 \\
\hline Hollow needle & $0.62(0.23-1.68)$ & $1.16(0.30-4.52)$ & .83 & $0.62(0.23-1.68)$ & $1.44(0.37-5.63)$ & .6 \\
\hline Instrument & $0.35(0.12-1.04)$ & $0.31(0.07-1.41)$ & .13 & $0.35(0.12-1.04)$ & $0.51(0.11-2.31)$ & .38 \\
\hline Needle, unknown type & $0.59(0.11-3.14)$ & $1.41(0.19-10.37)$ & .74 & $0.59(0.11-3.14)$ & $1.35(0.19-9.32)$ & .76 \\
\hline Other & $0.28(0.05-1.59)$ & $0.92(0.10-8.42)$ & .94 & $0.28(0.05-1.59)$ & $1.15(0.13-9.84)$ & .9 \\
\hline Splash & $0.17(0.07-0.43)$ & $0.17(0.03-1.11)$ & .06 & $0.17(0.07-0.43)$ & $0.18(0.03-1.20)$ & .08 \\
\hline \multicolumn{7}{|l|}{ Source rapid HIV test } \\
\hline Negative & Reference category & Reference category & & Reference category & Reference category & \\
\hline Positive & $0.02(0.01-0.07)$ & $0.02(0.004-0.13)$ & $<.01$ & $0.03(0.01-0.10)$ & $0.09(0.02-0.45)$ & $<.01$ \\
\hline Unknown & $0.07(0.03-0.13)$ & $0.03(0.01-0.08)$ & $<.01$ & $0.05(0.02-0.10)$ & $0.02(0.01-0.06)$ & $<.01$ \\
\hline \multicolumn{7}{|c|}{ Referral to infectious diseases department } \\
\hline No & Reference category & Reference category & & Reference category & Reference category & \\
\hline Yes & $0.15(0.08-0.32)$ & $0.41(0.12-1.39)$ & .15 & $0.15(0.08-0.32)$ & $0.12(0.04-0.37)$ & $<.01$ \\
\hline
\end{tabular}

$S P D$, sterile processing.

*Models include institution role, department, injury location, instrument type, source rapid HIV test, and infectious diseases referral.

\section{References}

1. Centers for Disease Control and Prevention. Sharps safety for healthcare settings. 2015. Available from: https://www.cdc.gov/sharpssafety/. Accessed April 25, 2016.

2. Pruss-Ustun A, Rapiti E, Hutin Y. Estimation of the global burden of disease attributable to contaminated sharps injuries among health-care workers. Am J Ind Med 2005;48:482-90.

3. Centers for Disease Control and Prevention. Updated U.S. Public Health Service guidelines for the management of occupational exposures to HBV, HCV, and HIV and recommendations for postexposure prophylaxis. MMWR Recomm Rep 2001:50(Rr-11):1-52.

4. Kuhar DT, Henderson DK, Struble KA, Heneine W, Thomas V, Cheever LW, et al. Updated US Public Health Service guidelines for the management of occupational exposures to human immunodeficiency virus and recommendations for postexposure prophylaxis. Infect Control Hosp Epidemiol 2013;34:875-92.

5. Advisory Committee on Immunization Practices. Immunization of health-care personnel: recommendations of the Advisory Committee on Immunization Practices (ACIP). MMWR Recomm Rep 2011;60(RR-7):1-45.

6. Gershon RR, Flanagan PA, Karkashian C, Grimes M, Wilburn S, Frerotte J, et al. Health care workers' experience with postexposure management of bloodborne pathogen exposures: a pilot study. Am J Infect Control 2000;28:421-8.

7. Ko NY, Yeh SH, Tsay SL, Pan SM, Feng MC, Chiang MC, et al. Adherence to management after occupational exposure to bloodborne pathogen among health care workers in Taiwan. Am J Infect Control 2009;37:609-11.

8. Tabak N, Shiaabana AM, Shasha S. The health beliefs of hospital staff and the reporting of needlestick injury. J Clin Nurs 2006;15:1228-39. 